\title{
Design of Wireless Power and Information Transfer Systems Considering Figure of Merit for Information
}

\author{
Gunyoung $\mathrm{Kim}^{1} \cdot$ Bomson Lee ${ }^{2, *}$
}

\begin{abstract}
Inductively coupled resonant wireless power transfer (WPT) systems can be used as a wireless power and information transfer (WPIT) system by properly adding the function of varying Rx loads. A new metric for the figure of merit for information transfer from Rx to Tx is proposed as the ratio of Tx input impedances for the $\mathrm{Rx}$ shorted and optimum loads to systematically assess the information transfer. While most of WPT and near-field communication (NFC) devices have been adopted for very short distances between Tx and Rx, this work shows that the WPIT systems using inductively coupled resonant structures with high Q-factor coils enable much longer working distances with the best power transfer efficiency and information transfer capability. Several design examples show that the newly proposed figure of merit for information transfer is an essential metric in the understanding and design of WPIT systems. The theory is validated with circuit and electromagnetic simulations for various system configurations.
\end{abstract}

Key Words: Figure of Merit for WPT, Magnetic Coupling, Near-Field Communication, Simultaneous Wireless Power and Information Transfer.

\section{INTRODUCTION}

Wireless power transfer (WPT) based on inductively coupled resonance was first introduced in [1], where an efficiency of about $40 \%$ was achieved with a separation of approximately $2 \mathrm{~m}$ between $\mathrm{Tx}_{\mathrm{x}}$ and Rx of spiral shapes. Since then, the inductively coupled resonant WPT technology has been developed in theory and also applied to many applications including WPTs to multiple receivers, electric mobile phones, home appliances, motor vehicles, biomedical devices, and so on [2-6]. In most practical WPT devices, the information of power-receiving units needs to be transferred to power-transmitting units. There may be a variety of information but the identification numbers, stored power levels, etc., are essentially required in the basic operation of WPT devices. To cope with this necessity, the simultaneous wireless and information power transfer (SWIPT) has been studied in various forms of geometry and for the possible merging of information and power in the near future [7-10]. However, most of the research papers in this area focus on the numerous architectures in the high level of the hierarchy. However, some papers focusing on actual structures enabling information transfer in the current WPT frame are at times available, under the name of wireless power and information transfer (WPIT).

In [11], theory of high-order modulation for near-field RF identification (RFID) and WPT was presented. A system that transmits power using a triangular current waveform and transmits information to its third-order harmonic was reported in

Manuscript received October 8, 2019 ; Revised December 24, 2019 ; Accepted April 9, 2020. (ID No. 20191008-085J)

${ }^{1} \mathrm{HW}$ Team (Radar), Hanwha Systems, Yongin, Korea.

${ }^{2}$ Department of Electronic Engineering, Kyung Hee University, Yongin, Korea.

"Corresponding Author: Bomson Lee (e-mail: bomson@khu.ac.kr)

This is an Open-Access article distributed under the terms of the Creative Commons Attribution Non-Commercial License (http://creativecommons.org/licenses/by-nc/4.0) which permits unrestricted non-commercial use, distribution, and reproduction in any medium, provided the original work is properly cited.

(c) Copyright The Korean Institute of Electromagnetic Engineering and Science. All Rights Reserved. 
[12]. In [13], a WPIT technology based on binary frequency shift keying modulation using the dual bands of a series-parallel combined resonant circuit was proposed. Its experimental prototype exhibited a communication rate up to $20 \mathrm{kbps}$, while maintaining a power transfer efficiency greater than $85 \%$ at a distance of $50 \mathrm{~mm}$. However, these systems are complex in configuration. In [14], a frequency-agile load modulation scheme was proposed for a reliable near-field magnetic resonance WPT system. A wireless power and bidirectional data transmission scheme through mutual coupling between two isolated LC resonators was proposed in [15]. The near-field communication (NFC) [16] used for RFID technology may also be promising for delivery of this information by means of load modulation techniques. The existing NFC and other WPIT demonstrations are practically limited within a distance of several centimeters.

In this paper, we use simple mathematical expressions to analyze a WPIT system in terms of power and information transfer capabilities. Then, we provide design equations for a specifically required efficiency and transfer capability of $\mathrm{Rx}$ status information. Especially, to assess the information (including Rx status) transfer capability from $\mathrm{Rx}$ to $\mathrm{Tx}_{\mathrm{x}}$ in a systematic manner, we propose a new metric using the input impedances at the $\mathrm{Tx}$ terminal depending on Rx loads. Its effectiveness and usefulness is validated by several design examples.

In Section II, the WPT performance of a single-input multiple-output (SIMO) system in terms of input impedances, efficiencies, and optimum loads for maximum efficiency are briefly discussed. Then, we newly define the figure of merit for information delivery using $\mathrm{Tx}$ input impedance variations depending on $\mathrm{Rx}$ load variations and demonstrate its usefulness. In Section III, we present some practical examples to validate the proposed WPIT methodology with electromagnetic (EM)-simulated results. The conclusions and closing comments are in Section IV.

\section{DESIGN THEORY}

Fig. 1 shows the equivalent circuit of a WPIT system. The system is composed of $\mathrm{Tx} 0$ (or $\mathrm{Tx}$ ) and $\mathrm{Rx} 1$ (or $\mathrm{Rx}$ ) coils (or loops), which may be a single turn loop or a multi-turn loop or a spiral coil, separated by a certain distance $d$. The loops, having inductances, are loaded with capacitors for LC resonance. The power is transferred from $\mathrm{Tx}$ to $\mathrm{Rx}$ via magnetically coupled resonant loops. $R_{i}$ and $L_{i}$ are the resistance and inductance of the $\operatorname{Tx}(i=0)$ and $\mathrm{Rx}(i=1)$ loops, respectively. $C_{i}$ is the capacitance of the lumped capacitor for $\operatorname{Tx}(i=0)$ and $\mathrm{Rx}(i=1)$, respectively. $r_{i}$ is the radius of the $i^{\text {th }}$ loop and $r_{i n, i}$ is the inner radius of the $i^{\text {th }}$ loop wire. $V_{0}$ is the voltage at the input. The singlepole single-through (SPST) switch is operated between off (for an optimum load) and on (for a shorted load). The usual func-

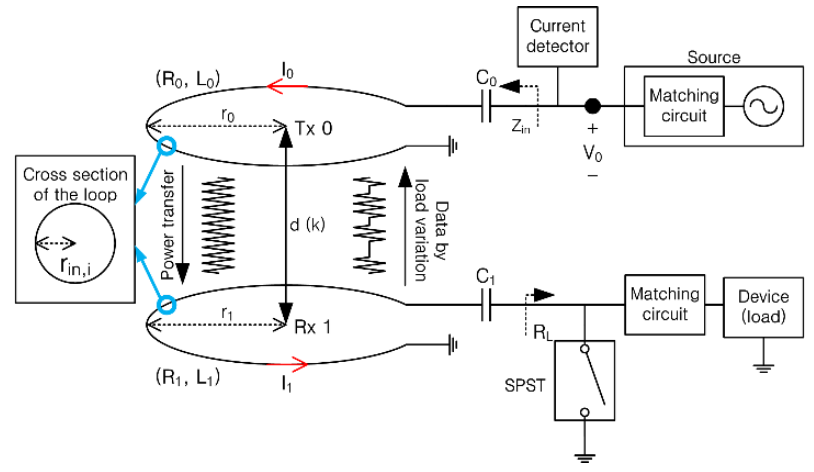

Fig. 1. Configuration of a magnetically coupled wireless power and information transfer (WPIT) system.

tion of the $\mathrm{Rx}$ matching circuit is to transform the usually small $\mathrm{Rx}$ optimum load to the large device load (say, $50 \Omega$ ). Conventional WPT has been made with the switch off to achieve maximum efficiency. The Rx status information (such as a device identity, energy charged levels, etc.) can be transferred from Rx to Tx by switching between the on and off states of the SPST. Based on this equivalent circuit, the system is usually formulated by a $Z$-matrix given by

$$
\begin{aligned}
& {\left[\begin{array}{c}
V_{0} \\
0
\end{array}\right]=\left[\begin{array}{ll}
Z_{00} & Z_{01} \\
Z_{10} & Z_{11}
\end{array}\right]\left[\begin{array}{c}
I_{0} \\
I_{1}
\end{array}\right]} \\
& =\left[\begin{array}{cc}
R_{0}+j \omega L_{0}+\frac{1}{j \omega C_{0}} & -j \omega k \sqrt{L_{0} L_{1}} \\
-j \omega k \sqrt{L_{0} L_{1}} & R_{1}+R_{L}+j \omega L_{1}+\frac{1}{j \omega C_{1}}
\end{array}\right]\left[\begin{array}{c}
I_{0} \\
I_{1}
\end{array}\right]
\end{aligned}
$$

where $k$ is the coupling coefficient between $\mathrm{Tx}$ and $\mathrm{Rx}$ loops [17]. It is well known that $k$ approaches 1 as the distance between $\mathrm{Tx}$ and $\mathrm{Rx}$ becomes 0 and it approaches 0 as the distance between $\mathrm{Tx}$ and $\mathrm{Rx}$ becomes very large. $V_{0}$ is the source voltage, $R_{L}$ is the $\mathrm{Rx}$ load resistance, and $\omega$ is the angular frequency. $I_{0}$ and $I_{1}$ are the currents on loop 1 and 2 , respectively. The ratio of currents on $\mathrm{Tx}$ and $\mathrm{Rx}$ loops is given by [18]:

$$
\frac{I_{1}}{I_{0}}=\frac{j \omega k \sqrt{L_{0} L_{1}}}{R_{1}+R_{L}+j \omega L_{1}+\frac{1}{j \omega C_{1}}} .
$$

It is observed that as $R_{L}$ increases, (2) becomes small and approaches 0 as $R_{L}$ goes to infinity (open). The phase of the current flowing on $\mathrm{Rx}\left(I_{1}\right)$ is $90^{\circ}$ ahead of that flowing on $\mathrm{Tx}\left(I_{0}\right)$ following Faraday's law. The input impedance is defined as the ratio of $V_{0}$ and $I_{0}$ on a Tx terminal and expressed as

$$
\begin{aligned}
& Z_{\text {in }}\left(\omega, R_{L}\right)=\frac{V_{0}}{I_{0}}=Z_{00}+Z_{01} \frac{I_{1}}{I_{0}} \\
& =R_{0}+j \omega L_{0}+\frac{1}{j \omega C_{0}}+\frac{(\omega k)^{2} L_{0} L_{1}}{R_{1}+R_{L}+j \omega L_{1}+\frac{1}{j \omega C_{1}}} .
\end{aligned}
$$


At the resonant frequency, the input impedance (3) reduces to

$$
Z_{\text {in }}\left(\omega_{0}, R_{L}\right)=R_{0}+\frac{\left(\omega_{0} k\right)^{2} L_{0} L_{1}}{R_{1}\left(1+R_{L} / R_{1}\right)} .
$$

If we define the figure of merit for the power transfer $(F)$ as

$$
F=k \sqrt{Q_{0} Q_{1}}=k Q\left(Q_{0}=\frac{\omega_{0} L_{0}}{R_{0}}, Q_{1}=\frac{\omega_{0} L_{1}}{R_{0}}\right),
$$

the input impedance (4) at the resonant frequency depending on the $\mathrm{Rx}$ load $R_{L}$ can be re-written as

$$
Z_{\text {in }}=R_{0}\left(1+\frac{F^{2}}{1+R_{L} / R_{1}}\right),
$$

which is a purely real number. At this point, the essential part of power transfer efficiency for a resonant WPT system needs to be summarized [17]. The efficiency can be stated in a simple manner by

$$
\eta(F, b)=\frac{F^{2}}{\left(1+b \sqrt{1+F^{2}}\right)\left(1+\frac{1}{b} \sqrt{1+F^{2}}\right)},
$$

where $b$ is the deviation factor against the Rx optimum load $R_{L, o p t}$ defined by

$$
b=\frac{R_{L}}{R_{L, o p t}}=\frac{R_{L}}{R_{1} \sqrt{1+F^{2}}} .
$$

A short observation of (7) tells that it is a function of the figure of merit for power transfer $(F)$ and $b$, and a large $F$ is merely a necessary condition for a high efficiency. The maximum efficiency is guaranteed with the additional condition of $b=1$ $\left(R_{L}=R_{L, o p t}=R_{1} \sqrt{1+F^{2}}\right)$.

Now, we examine the ways of transferring $\mathrm{Rx}$ status information (or others) to Tx. Monitoring (6) at the Tx terminal depending on $R_{L}$ is just like monitoring the current on Tx $\left(I_{0}\right)$ depending on $R_{L}$ since $I_{0}=V_{0} / Z_{i n}$. When $R_{L}=0$ (short), the system is at the over-coupled limit, no power is transferred to $\mathrm{Rx}$, and the efficiency (7) is 0 . However, the largest current is induced on $\mathrm{Rx}$ and the magnetic flux back to Tx affects the $\mathrm{Tx}$ input impedance most significantly, and the input impedance becomes the largest: $R_{0}\left(1+F^{2}\right)$. With this, the Tx current $I_{0}$ becomes the smallest. When $R_{L}=\infty$ (open), no current is induced on the $\mathrm{Rx}$ loop, the system is at the under-coupled limit, no power is transferred to $R x$, the efficiency (7) is also 0 , the $T x$ is as if being isolated from $\mathrm{Rx}$, and the Tx input impedance becomes that of its own: $R_{0}$. When $R_{L}=R_{L, o p t}=R_{1} \sqrt{1+F^{2}} \quad(b=$ 1) (optimum for maximum efficiency), (6) becomes

$$
Z_{\text {in }}=R_{0}\left(1+\frac{F^{2}}{1+\sqrt{1+F^{2}}}\right)=R_{0} \sqrt{1+F^{2}} .
$$

Table 1. Input impedances and transfer efficiencies depending on $\mathrm{Rx}$ loads at resonant frequency

\begin{tabular}{lcccc}
\hline \multicolumn{1}{c}{ Load status } & $R_{L}(\Omega)$ & $b$ & $Z_{\text {in }}\left(\omega_{0}\right)(\Omega)$ & $\eta\left(\omega_{0}\right)$ \\
\hline $\begin{array}{l}\text { Short (over- } \\
\text { coupled limit) }\end{array}$ & 0 & 0 & $R_{0}\left(1+F^{2}\right)$ & 0 \\
$\begin{array}{l}\text { Optimum (crit- } \\
\text { ically-coupled) }\end{array}$ & $R_{1} \sqrt{1+F^{2}}$ & 1 & $R_{0} \sqrt{1+F^{2}}$ & $\frac{F^{2}}{\left(1+\sqrt{1+F^{2}}\right)^{2}}$ \\
$\begin{array}{l}\text { Open (under- } \\
\text { coupled limit) }\end{array}$ & $\infty$ & $\infty$ & $R_{0}$ & 0 \\
\hline
\end{tabular}

The current variations on $\mathrm{Tx}$ (or the input impedance variations) depending on $R_{L}$ (open, short, optimum) can be used for the information transfer from $\mathrm{Rx}$ to $\mathrm{Tx}$. These input impedances (6) and the power transfer efficiencies (7) are summarized in Table 1.

NFC [16] more or less utilizes these variations of $Z_{\text {in }}$ at the Tx terminal occurring between the two load states of $\mathrm{Rx}$, but mostly at a close proximity of $\mathrm{Tx}$ to $\mathrm{Rx}$. Using the results in Table 1, we can assess the transfer capability of Rx information for the usual resonant WPT systems.

The best choice of the Rx load set for information transfer from $\mathrm{Rx}$ to Tx may be the short (over-coupled limit) and open (under-coupled limit) states, since this results in the largest current variation at the $\mathrm{Tx}$ with the effect of a lowest bit error rate. However, it is noted that with this choice, power is not transferred to Rx. The best Rx load choice for WPIT may be the short (for the greatest magnetic flux effect to $\mathrm{Tx}$ ) and optimum (for the highest power transfer efficiency) loads. The real resistance of the SPST switch (Fig. 1) for the short state is not exactly 0 . The practical resistance is about $10 \mathrm{~m} \Omega$ but does not much affect the results in Table 1.

For the purpose of quantitative assessment by a new metric, we define the figure of merit for $\mathrm{Rx}$ information transfer to $\mathrm{Tx}$ $\left(F_{I}\right)$ as a ratio of the $\mathrm{Tx} Z_{\text {in }}$ when $R_{L}=0$ and the same when $R_{L}$ $=R_{L, o p t}$ in Table 1 , given by

$$
F_{I}=\frac{Z_{\text {in }}\left(R_{L}=0\right)}{Z_{\text {in }}\left(R_{L}=R_{L, \text { opt }}\right)}=\sqrt{1+F^{2}} .
$$

The figure of merit for $\mathrm{Rx}$ information transfer to $\mathrm{Tx}\left(F_{I}\right)$ as defined in (10) also corresponds to the current $\left(I_{0}\right)$ variation at the Tx terminal depending on Rx loads of "short" and "optimum" $R_{L}$ since $I_{0}=V_{0} / Z_{\text {in }}$. The metric (10) is plotted in Fig. 2 together with the maximum efficiencies as a function of the figure of merit for power transfer $(F)$. We can see that as the figure of merit for power $(F)$ increases, the figure of merit for information transfer $\left(F_{I}\right)$ also increases and the power transfer efficiency also increases. Notice that when $F$ is roughly larger than 3 , the efficiency is greater than $50 \%$ and $F_{I}$ almost ap- 


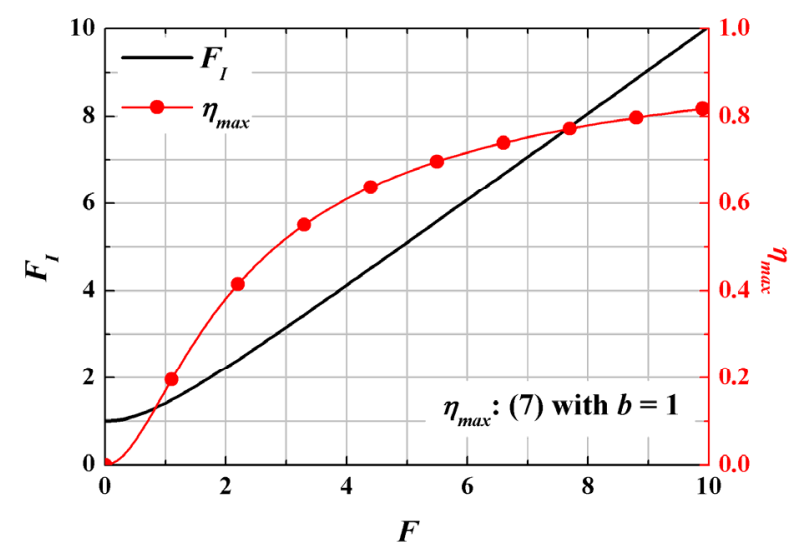

Fig. 2. Figure of merit for $\mathrm{Rx}$ information transfer to $\mathrm{Tx}\left(F_{I}\right)$ and maximum efficiency $\left(\eta_{\max }\right)$ at the resonant frequency as a function of figure of merit for power $(F)$ for a WPIT system.

proaches $F$. Taking an example, the transfer efficiency of about $80 \%$ is achieved with $F=9$, where $F_{I}$ is also about 9 .

\section{DESIGN EXAMPLES VALIDATED WITH}

\section{ELECTROMAGNETIC SIMULATIONS}

The real resonant WPT systems realized for an $F$ given by (5) are numerous. Several realizations for a system consisting of a Tx loop with $r_{0}=10 \mathrm{~cm}$ and a Rx loop with $r_{0}=5 \mathrm{~cm}$ are shown in Table 2, where the quality factor $Q$ is the geometrical mean of $Q_{0}$ and $Q_{1}(5)$. The coupling coefficients $k$ were obtained using [16].

In Table 3, we summarize the same as in Table 2 but for a different system with larger Tx and Rx loops with $r_{0}=r_{1}=20 \mathrm{~cm}$, enabling power and information transfer in longer ranges. It is seen in Table 3 that an efficiency of $56 \%$ is achieved for the case

Table 2. Examples of design system configurations $\left(r_{0}=10 \mathrm{~cm}, r_{1}\right.$ $=5 \mathrm{~cm}, r_{i n 0}=r_{i n 1}=0.1 \mathrm{~cm}, Q_{1}=388.81$, and $\left.Q_{2}=334.23\right)$

\begin{tabular}{cccccc}
\hline$d(\mathrm{~cm})$ & $k$ & $Q$ & $F$ & $F_{I}$ & $\eta_{\max }(\%)$ \\
\hline 5 & 0.0904 & 360.5 & 32.6 & 32.6 & 94.0 \\
10 & 0.0419 & 360.5 & 15.1 & 15.1 & 87.6 \\
15 & 0.0203 & 360.5 & 7.3 & 7.4 & 76.1 \\
20 & 0.0108 & 360.5 & 3.9 & 4.0 & 60.2 \\
25 & 0.0063 & 360.5 & 2.3 & 2.5 & 42.4 \\
30 & 0.0039 & 360.5 & 1.4 & 1.7 & 26.7 \\
\hline
\end{tabular}

Table 3. Examples of design system configurations $\left(r_{0}=r_{1}=20 \mathrm{~cm}\right.$,

\begin{tabular}{cccccc}
\multicolumn{5}{c}{$\left.r_{i n 0}=r_{i n 1}=0.2 \mathrm{~cm}, Q_{0}=Q_{1}=776.2\right)$} \\
\hline$d(\mathrm{~cm})$ & $k$ & $Q$ & $F$ & $F_{I}$ & $\eta_{\max }(\%)$ \\
\hline 10 & 0.1882 & & 146.1 & 146.1 & 98.6 \\
20 & 0.0836 & & 64.9 & 64.9 & 97.0 \\
40 & 0.0240 & & 18.6 & 18.7 & 89.8 \\
60 & 0.0093 & 776.2 & 7.2 & 7.3 & 75.9 \\
80 & 0.0044 & & 3.4 & 3.6 & 56.1 \\
100 & 0.0024 & & 1.9 & 2.1 & 35.6 \\
\hline
\end{tabular}

of $d=80 \mathrm{~cm}$ with $F_{I} \approx F=7.3$. The figure of merit for power $(F)$, one important parameter in the resonant WPT efficiency, can now be understood as the $\mathrm{Tx}$ current variations used for $\mathrm{Rx}$ information transfer.

We take the two system configurations with $d=15 \mathrm{~cm}$ and $25 \mathrm{~cm}$ in Table 2 and plot the real part (a) and imaginary part (b) of the input impedance (3) for different loads of $R_{L}=0$ and $R_{L}=R_{L, o p t}=R_{1} \sqrt{1+F^{2}}$ as a function of frequency in Fig. 3 . The radii of $\mathrm{Tx}$ and $\mathrm{Rx}$ loops are $10 \mathrm{~cm}\left(Q_{0}=388.8\right)$ and $5 \mathrm{~cm}$ $\left(Q_{1}=334.2\right)$, respectively. The $\mathrm{Tx}$ and $\mathrm{Rx}$ loop wire radii are $0.1 \mathrm{~cm}$. The imaginary parts (b) of the input impedances for both cases of $d=15 \mathrm{~cm}$ and $d=25 \mathrm{~cm}$ are observed to be zero at the resonant frequency since the system is at resonance. The real parts (a) of the input impedances at the resonant frequency, given by (6) and in Table 1 , have the maximums when $R_{L}=0$. The circuit-simulated figures of merit for information transfer $\left(F_{I}\right)$ are 3.7/0.5 (=7.38) for the case of $d=15 \mathrm{~cm}$ and $0.42 / 0.17$ $(=2.48)$ for the case of $d=25 \mathrm{~cm}$. The EM-simulated figures

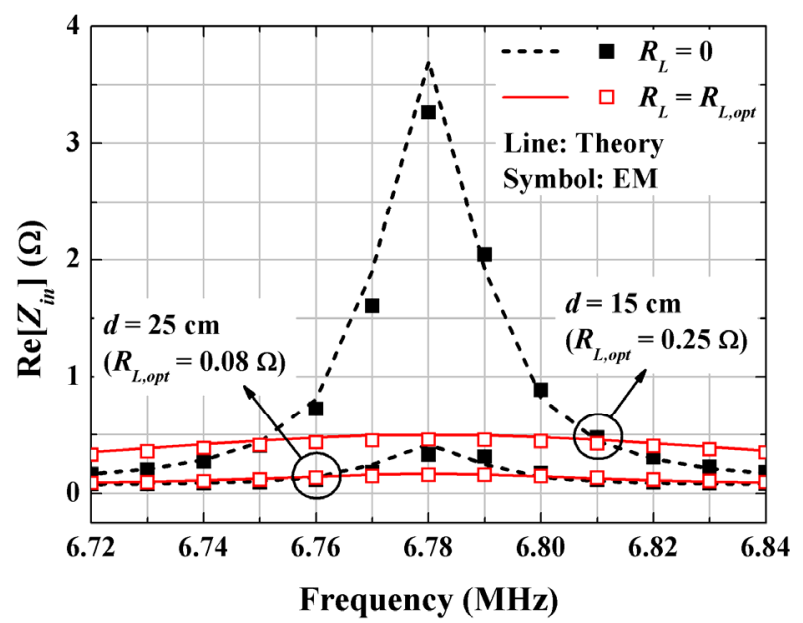

(a)

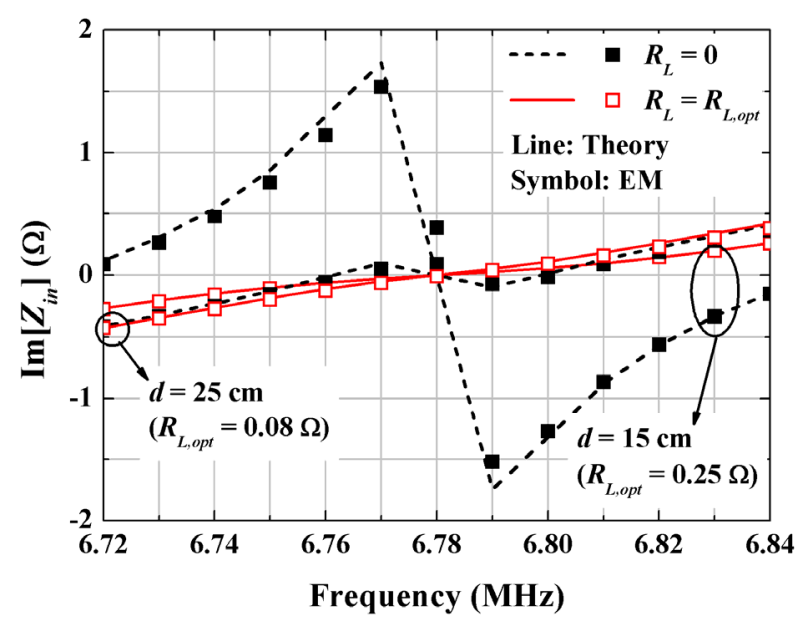

(b)

Fig. 3. Input impedances of SISO system for different loads $\left(r_{0}=\right.$ $10 \mathrm{~cm}, r_{1}=5 \mathrm{~cm}, r_{i n 0}=r_{i n 1}=0.1 \mathrm{~cm}, Q_{1}=388.81$, and $Q_{2}=$ 334.23): (a) real parts and (b) imaginary parts. 
of merit for information transfer $\left(F_{I}\right)$ are $3.26 / 0.45(=7.2)$ for the case of $d=15 \mathrm{~cm}$ and $0.33 / 0.16(=2.1)$ for the case of $d=$ $25 \mathrm{~cm}$. The results are in agreement with the theoretical ones of 7.4 and 2.5 in Table 2. These $F_{I}$ s should be understood as the Tx current variations due to the $\mathrm{Rx}$ load changes. The overall EM-simulated input impedances are shown to be in good agreement with the theoretical ones.

Fig. 4 shows the transient responses of the Tx currents when the Rx load switches between with $R_{L}=0$ and $R_{L}=R_{L, o p t}=$ $R_{1} \sqrt{1+F^{2}}$ with $10 \mathrm{kbps}(\mathrm{a})$ and $20 \mathrm{kbps}(\mathrm{b})$ for the case of $d=$ $15 \mathrm{~cm}$ and $Q=\sqrt{Q_{0} Q_{1}}=360.5$ in Table 2. Based on the $\mathrm{AC}$ steady state analysis, the Tx current variation (characterized by $\left.F_{I}\right)$ with each $\mathrm{Rx}$ load has been found to be almost identical to the theoretical value of 7.4. Based on the transient analysis with increasing switching rates, Fig. 4(a) and 4(b) show that it takes about $0.5 \mathrm{~ms}$ for the Tx current to reach a steady state. The source voltage $V_{0}$ in Fig. 1 is assumed to be $1 \mathrm{~V}$. For the shorted Rx load with $R_{L}=0 \Omega$ and optimum load with $R_{L}=0.25 \Omega$, the average Tx current envelopes are $0.33 \mathrm{~A}$ and $1.97 \mathrm{~A}$, respectively, for the case of the 10 kbps switching rate (Fig. 4(a)). Thus,

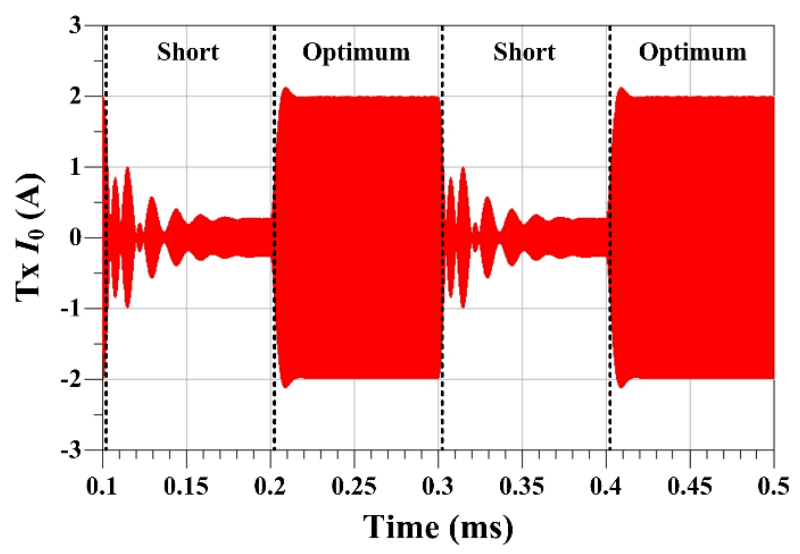

(a)

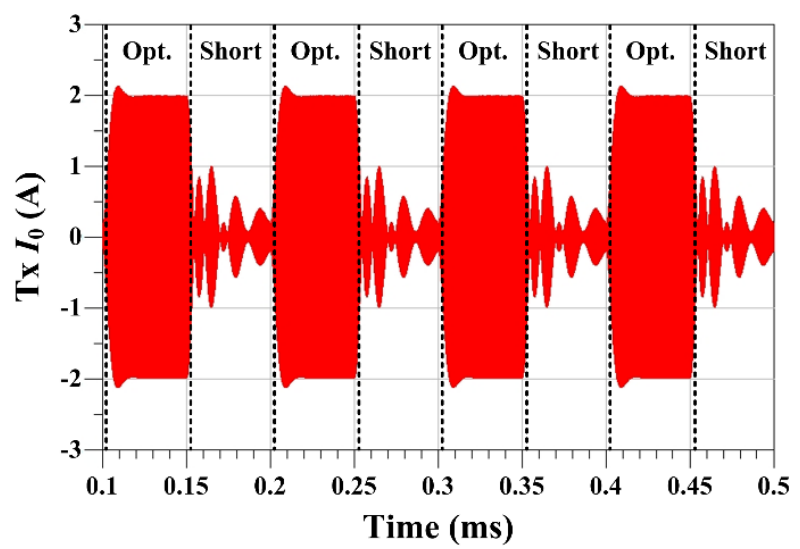

(b)

Fig. 4. Circuit-simulated transient $\mathrm{Tx}$ current $\left(I_{0}\right)$ for different $\mathrm{Rx}$ loads with 0 (short) and $0.25 \Omega$ (optimum) $\left(V_{0}=1 \mathrm{~V}\right.$, frequency $=6.78 \mathrm{MHz}, r_{0}=10 \mathrm{~cm}, r_{1}=5 \mathrm{~cm}, r_{i n 0}=r_{i n 1}=0.1$ $\mathrm{cm}, Q_{1}=388.8, Q_{2}=334.2, d=15 \mathrm{~cm}, k=0.0203$, and $F$ $=7.3$ ): (a) data rate $=10 \mathrm{kbps}$ and (b) data rate $=20 \mathrm{kbps}$.

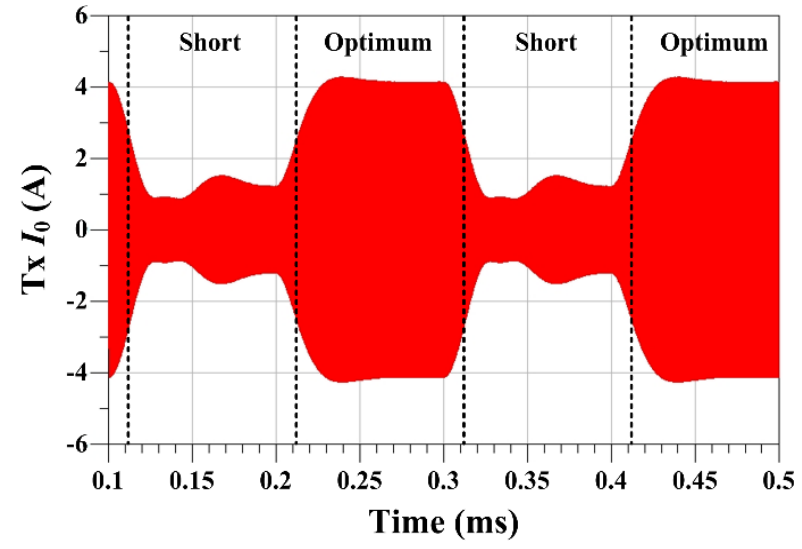

(a)

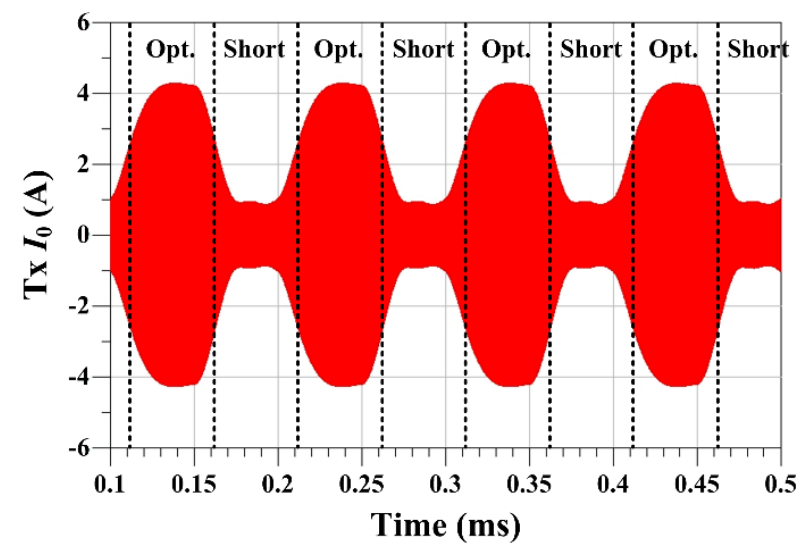

(b)

Fig. 5. Circuit-simulated transient Tx current $\left(I_{0}\right)$ for different $\mathrm{Rx}$ loads with 0 (short) and $0.24 \Omega$ (optimum) $\left(V_{0}=1 \mathrm{~V}\right.$, frequency $=6.78 \mathrm{MHz}, r_{0}=r_{1}=20 \mathrm{~cm}, r_{i n 0}=r_{i n 1}=0.2 \mathrm{~cm}, Q_{1}$ $=Q_{2}=776.2, d=80 \mathrm{~cm}, k=0.0044$, and $F=3.4$ ): (a) data rate $=10 \mathrm{kbps}$ and (b) data rate $=20 \mathrm{kbps}$.

the ratio of the Tx currents is $6.0(=1.97 / 0.33)$. For the case of $20 \mathrm{kbps}$ (Fig. 4(b)), the ratio of the Tx current envelopes is 5.0 $(=1.96 / 0.39)$. Even though the ratio of Tx currents somewhat deviates from the expected $F_{I}$ due to the effects of the transients from switching operations.

Fig. 5 shows the same as plotted in Fig. 4 for the different configuration of $d=80 \mathrm{~cm}$ and $Q=\sqrt{Q_{0} Q_{1}}=776.2(F=3.4$, $\left.F_{I}=3.6\right)$ in Table 3. The Tx current variation with the mentioned two $\mathrm{Rx}$ loads when reached in steady state is about 3.5 (close to the theoretical $F_{I}$ of 3.6). The source voltage $V_{0}$ in Fig. 1 is assumed to be $1 \mathrm{~V}$. The short and optimum loads $\left(R_{L} \mathrm{~s}\right)$ are $0 \Omega$ and $0.24 \Omega$. The ratio of Tx current envelopes is 3.3 for the case of $10 \mathrm{kbps}$ and 3.1 for the case of $20 \mathrm{kbps}$, again close to the theoretical value $\left(F_{I}\right)$ of 3.6 .

The figure of merit for information transfer $\left(F_{I}\right)$ as defined in (10), expressed as a function of the figure of merit for power transfer $(F)$, has been shown to be very useful in characterizing and estimating the resonant WPIT systems, especially in their capability of transferring $\mathrm{Rx}$ information to $\mathrm{Tx}$. 


\section{CONCLUSION}

A new metric named "a figure of merit for information transfer" has been defined to assess WPIT systems in their capability to transfer information from $\mathrm{Rx}$ to $\mathrm{Tx}$. Its usefulness has been verified for several WPIT system configurations with wide ranges of $\mathrm{Tx}$ to $\mathrm{Rx}$ distances. It has been found to be close to the figure of merit for power and shown to be the ratio of the Tx currents when the Rx load is shorted and optimum. Introduction of the new metric is expected to put a basic foundation in the evaluation of WPIT systems which started being developed recently.

This research was supported by the Ministry of Science, ICT and Future Planning, Korea, under the Information Technology Research Center support program (No. IITP2020-2016-0-00291) supervised by the IITP (National IT Industry Promotion Agency).

\section{REFERENCES}

[1] Kurs, R. Moffatt, and M. Soljacic, "Simultaneous mid-range power transfer to multiple devices," Applied Physics Letters, vol. 96, no. 4, article no. 044102, 2010.

[2] S. Kim, S. Hwang, S. Kim, and B. Lee, "Investigation of single-input multiple-output wireless power transfer systems based on optimization of receiver loads for maximum efficiencies," Journal of Electromagnetic Engineering and Science, vol. 18, no. 3, pp. 145-153, 2018.

[3] S. Cheon, Y. H. Kim, S. Y. Kang, M. L. Lee, J. M. Lee, and T. Zyung, "Circuit-model-based analysis of a wireless energytransfer system via coupled magnetic resonances," IEEE Transactions on Industrial Electronics, vol. 58, no. 7, pp. 29062914, 2011.

[4] A. K. RamRakhyani, S. Mirabbasi, and M. Chiao, "Design and optimization of resonance-based efficient wireless power delivery systems for biomedical implants," IEEE Transactions on Biomedical Circuits and Systems, vol. 5, no. 1, pp. 4863, 2011.

[5] J. H. Choi, S. K. Yeo, S. Park, J. S. Lee, and G. H. Cho, "Resonant regulating rectifiers (3R) operating for $6.78 \mathrm{MHz}$ resonant wireless power transfer (RWPT)," IEEE Journal of Solid-State Circuits, vol. 48, no. 12, pp. 2989-3001, 2013.

[6] Y. Zhang, T. Lu, Z. Zhao, F. He, K. Chen, and L. Yuan, "Selective wireless power transfer to multiple loads using receivers of different resonant frequencies," IEEE Transactions on Power Electronics, vol. 30, no. 11, pp. 6001-6005, 2015.

[7] I. Krikidis, S. Timotheou, S. Nikolaou, G. Zheng, D. W. K. $\mathrm{Ng}$, and R. Schober, "Simultaneous wireless information and power transfer in modern communication systems," IEEE Communications Magazine, vol. 52, no. 11, pp. 104-110, 2014.

[8] C. F. Liu, M. Maso, S. Lakshminarayana, C. H. Lee, and T. Q. Quek, "Simultaneous wireless information and power transfer under different CSI acquisition schemes," IEEE Transactions on Wireless Communications, vol. 14, no. 4, pp. 1911-1926, 2015.

[9] T. D. P. Perera, D. N. K. Jayakody, S. K. Sharma, S. Chatzinotas, and J. Li, "Simultaneous wireless information and power transfer (SWIPT): recent advances and future challenges," IEEE Communications Surveys \& Tutorials, vol. 20, no. 1, pp. 264-302, 2018.

[10] D. K. P. Asiedu, S. Mahama, S. W. Jeon, and K. J. Lee, "Optimal power splitting for simultaneous wireless information and power transfer in amplify-and-forward multiple-relay Systems," IEEE Access, vol. 6, pp. 3459-2468, 2018.

[11] J. Besnoff, M. Abbasi, and D. S. Ricketts, "High data-rate communication in near-field RFID and wireless power using higher order modulation," IEEE Transactions on Microwave Theory and Techniques, vol. 64, no. 2, pp. 401-413, 2016.

[12] Z. Yan, Z. Xiang, L. Wu, and B. Wang, "Study of wireless power and information transmission technology based on the triangular current waveform," IEEE Transactions on Power Electronics, vol. 33, no. 2, pp. 1368-1377, 2018.

[13] J. G. Kim, G. Wei, M. H. Kim, H. S. Ryo, and C. Zhu, "A wireless power and information simultaneous transfer technology based on 2FSK modulation using the dual bands of series-parallel combined resonant circuit," IEEE Transactions on Power Electronics, vol. 34, no. 3, pp. 29562965, 2018.

[14] N. Y. Kim, S. W. Yoon, and C. W. Kim, "Frequency-agile load-modulated magnetic resonance wireless power transfer system for reliable near-field in-band signalling," Electronics Letters, vol. 49, no. 24, pp. 1558-1559, 2013.

[15] C. C. Huang and C. L. Lin, "Wireless power and bidirectional data transfer scheme for battery charger," IEEE Transactions on Power Electronics, vol. 33, no. 6, pp. 46794689, 2018.

[16] J. I. Cairo, J. Bonache, F. Paredes, and F. Martin, "Reconfigurable system for wireless power transfer (WPT) and near field communications (NFC)," IEEE Journal of Radio Frequency Identification, vol. 1, no. 4, pp. 253-259, 2017.

[17] G. Kim and B. Lee, "Alternative expressions for mutual inductance and coupling coefficient applied in wireless power transfer," Journal of Electromagnetic Engineering and Science, vol. 16, no. 2, pp. 112-118, 2016.

[18] G. Kim, S. Boo, S. Kim, and B. Lee, "Control of power distribution for multiple receivers in SIMO wireless power transfer system," Journal of Electromagnetic Engineering and Science, vol. 18, no. 4, pp. 221-230, 2018. 


\section{Gunyoung Kim}

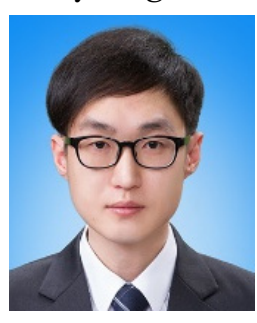

received a B.S. in Radio Communication Engineering from Kyung Hee University, Yongin, Korea in 2010, an M.S. in Electronics and Radio Engineering from Kyung Hee University in 2012, and a Ph.D. in Electronic Engineering from Kyung Hee University in 2018. He is currently working at Hanwha Systems. His fields of research include microwave antennas, phased array antenna, and radar systems.

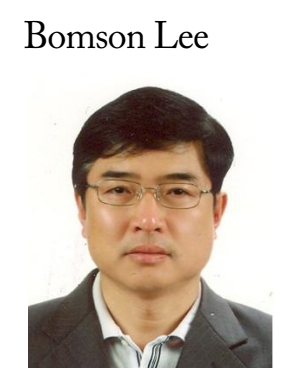

received the B.S. degree in Electrical Engineering from Seoul National University, Seoul, Korea, in 1982. From 1982 to 1988, he was with the Hyundai Engineering Company Ltd., Seoul, Korea. He received the M.S. and Ph.D. degrees in Electrical Engineering from the University of Nebraska, Lincoln, NE, USA, in 1991 and 1995, respectively. In 1995, he joined the faculty at Kyung Hee University, where he is currently a professor in the Department of Electronic Engineering. From 2007 to 2008, he was the chair of the technical group for microwave and radio wave propagation in the Korea Institute of Electromagnetic Engineering \& Science (KIEES). In 2010, he was an editor-inchief of the Journal of the Korean Institute of Electromagnetic Engineering and Science. In 2018, he served as the president of KIEES. His research activities include microwave antennas, RF identification (RFID) tags, microwave passive devices, wireless power transfer, and metamaterials. 